\title{
COVID-19 als Berufskrankheit oder Arbeitsunfall: Überlegungen zu Versicherungsschutz und Meldepflicht in der gesetzlichen Unfallversicherung
}

\section{COVID-19 as an occupational disease or work-related accident: Considerations regarding insurance cover and reporting obligation in the statutory accident insurance}

Autoren

Dennis Nowak ${ }^{1,2}$, Uta Ochmannn, ${ }^{1,}$, Stephan Brandenburg ${ }^{3}$, Albert Nienhaus ${ }^{3,4}$, Michael Woltjen ${ }^{3}$

Institute

1 Institut und Poliklinik für Arbeits-, Sozial- und Umweltmedizin, LMU, Klinikum München

2 Comprehensive Pneumology Center (CPC) München, Mitglied im Deutschen Zentrum für Lungenforschung (DZL)

3 Berufsgenossenschaft für Gesundheitsdienst und Wohlfahrtspflege (BGW) Hamburg

4 Kompetenzzentrum für Epidemiologie und Versorgungsforschung bei Pflegeberufen (CVcare), Universitätsklinikum Hamburg-Eppendorf

Schlüsselwörter

COVID-19-Erkrankung, Berufskrankheit, Arbeitsunfall

Key words

COVID-19 disease, occupational disease, occupational accident

online publiziert 04.01 .2021

Bibliografie

Dtsch Med Wochenschr 2021; 146: 198-204

DOI 10.1055/a-1341-7867

ISSN 0012-0472

(c) 2021. Thieme. All rights reserved.

Georg Thieme Verlag KG, Rüdigerstraße 14,

70469 Stuttgart, Germany

Korrespondenzadresse

Prof. Dr. med. Dennis Nowak

Institut und Poliklinik für Arbeits-, Sozial- und Umweltmedizin LMU Klinikum, Ziemssenstr. 1, 80336 München, Deutschland Tel.: $+49 / 89 / 440052301$

Fax: $+49 / 89 / 54445$

d.nowak@lmu.de

\section{ZUSAMMENFASSUNG}

Eine COVID-19-Erkrankung kann Berufskrankheit oder Arbeitsunfall sein. Eine Anerkennung als Berufskrankheit
3101 kann erfolgen, wenn die Erkrankung bei versicherten Personen auftritt, die infolge der Ausübung ihrer beruflichen Tätigkeit in einem von vier Bereichen einer gegenüber der Allgemeinbevölkerung wesentlich erhöhten Infektionsgefahr ausgesetzt waren: (1) Gesundheitsdienst, (2) Wohlfahrtspflege, (3) Laboratorium oder (4) bei anderer Tätigkeit mit ähnlich wie bei (1) bis (3) erhöhter Infektionsgefahr. Der Versicherungsschutz bezieht sich auf Arbeitnehmerinnen und Arbeitnehmer, Selbständige - soweit nicht befreit - und Ehrenamtliche. Gesetzlich meldepflichtig ist die COVID19-Erkrankung, meist in Verbindung mit zeitnahem SARSCoV-2-Erregernachweis. Eine COVID-19-Erkrankung kann dann als Arbeitsunfall vorliegen, wenn sich der intensive und direkte Kontakt zu infizierten Personen nicht bestimmungsgemäß wie bei der Berufskrankheit 3101, sondern anderweitig situativ aus der versicherten Tätigkeit ergibt.

\section{ABSTRACT}

The COVID-19 illness can occur as an occupational disease or work-related accident. According to the German list of occupational diseases, recognition as an occupational disease 3101 requires occupational exposure of an insured person who has been exposed to an increased risk of infection compared to the general population as a result of their occupational activity in one of the four areas: (1) health service or (2) social welfare sector, (3) laboratory or (4) during activities with increased risk of infection comparable to (1) to (3). The insurance cover covers employees, self-employed people - if not exempted from insurance cover - and honorary workers. The COVID-19 disease is subject to legal notification, mostly in conjunction with a contemporary SARS-CoV-2 virus detection. Regarding insured people who are not included within the aforementioned areas (1) to (4), the COVID-19 illness can be acknowledged as an occupational accident if the intense and direct contact with infected people - not intended as in the case of occupational disease 3101 - but otherwise situationally results from the insured activity itself. 


\section{Einführung}

Ausbrüche von Epidemien bedingen ein erhöhtes Infektionsrisiko für Personen, die im Gesundheitsdienst und in der Wohlfahrtspflege arbeiten [1, 2]. Das hat sich bei den ersten beiden Corona-Epidemien SARS und MERS gezeigt und wird auch bei der COVID-19 Pandemie wieder so sein, wie ein exponentieller Anstieg der COVID-19 Fälle bei Beschäftigten im britischen Gesundheitswesen (NHS) bis zur Einführung von Maßnahmen zur sozialen Distanzierung belegt [3]. Erste Publikationen aus China und Italien sprechen dafür, dass der Anteil der Ärzte und Pflegekräfte unter den Infizierten überproportional erhöht ist [4-6]. Allerdings spricht die Erfahrung aus Singapur dafür, dass Beschäftigte im Gesundheitswesen effektiv vor SARS-CoV-2 geschützt werden können, wenn die notwendige Schutzausrüstung zur Verfügung steht [7]. Deshalb ist eine differenzierte Betrachtung des beruflichen Infektionsrisikos notwendig. Zur Betroffenheit der Beschäftigten im deutschen Gesundheitswesen können derzeit noch keine abschließenden Aussagen gemacht werden $[8,9]$. Nach dem täglichen Lagebericht des Robert Koch-Instituts zur Coronavirus-Krankheit-2019 vom 14.12.2020 wurden den Gesundheitsämtern bisher 35.042 Infektionen von Menschen gemeldet, die in Krankenhäusern, ärztlichen Praxen, Dialyseeinrichtungen und im Rettungsdienst tätig sind [10]. Nicht in allen Fällen hatten sich die Betroffenen im beruflichen Umfeld infiziert. Der Berufsgenossenschaft für Gesundheitsdienst und Wohlfahrtspflege (BGW), die u. a. auch für nichtstaatliche und kirchliche Unternehmen des Gesundheitsdienstes der zuständige Unfallversicherungsträger ist, wurden bis zum 11.12.2020 insgesamt 8.677 bestätigte SARS-CoV-2 Infektionen bei Beschäftigten aus (zahn-)ärztlichen Praxen und Kliniken gemeldet. Wird berücksichtigt, dass von den jährlich bei allen Unfallversicherungsträgern gemeldeten Infektionen etwa die Hälfte auf die BGW entfällt, ist zu vermuten, dass ein großer Teil der vom RKI erfassten Fälle bei Beschäftigten beruflich verursacht wurde [11].

Die Mortalität für COVID-19-Erkrankte bei den Berufstätigen beträgt entsprechend der Daten des RKI 0,15\% [10]. Diese Schätzung wird durch die Meldungen bei der BGW bestätigt. Auch hier beträgt die Mortalität bei Versicherten mit einer SARS-CoV-2-Infektion $0,13 \%$. Auch wenn diese Daten die Mortalität aufgrund nicht detektierter Fälle wahrscheinlich überschätzen, so sprechen sie in Kombination mit pneumologischen (z. B. Lungenfibrosen), kardiologischen (z. B. Herzmuskelschädigungen), neurologischen (Einschränkungen des Geruchs- und Geschmackssinns) und weiteren Spätfolgen, die nach heutigem begrenztem Wissensstand vorstellbar erscheinen, dafür, dass die versicherungsrechtlichen Auswirkungen erheblich sein können - auch wenn es sich in der Mehrzahl der Fälle um ein leichtes und passageres Krankheitsbild handelt.

Im Zusammenhang mit der aktuellen Situation ergeben sich in Bezug auf die gesetzliche Unfallversicherung für die Ärzteschaft zwei wesentliche Fragestellungen, die durch diesen Beitrag beantwortet werden sollen. Ärztinnen und Ärzte sind - wie auch viele andere Berufsgruppen, die im medizinischen und pflegerischen Bereich arbeiten - aufgrund ihrer beruflichen Tätigkeit einem erhöhten Infektionsrisiko ausgesetzt. Im ersten Teil dieses Beitrags soll daher zunächst dargestellt werden, ob und in welchem Umfang die genannten Berufsgruppen bei ihrer Arbeit unter dem
Schutz der Unfallversicherung stehen. Ein besonderer Schwerpunkt liegt dabei auf der Darstellung, unter welchen rechtlichen Voraussetzungen COVID-19 als Berufskrankheit bei ihnen anerkannt werden kann. In seinem zweiten Teil befasst sich dieser Artikel mit der Frage, in welchen Fällen Ärztinnen und Ärzte ihrer gesetzlichen Pflicht zur Meldung einer Berufskrankheit nachkommen müssen, wenn sie im Rahmen der Behandlung den Verdacht haben, eine Patientin / ein Patient könnte sich möglicherweise in Ausübung der beruflichen Tätigkeit mit dem Corona-Virus infiziert haben. Der dritte und letzte Abschnitt befasst sich mit der Frage, ob und ggf. unter welchen Voraussetzungen COVID-19 die Kriterien eines Arbeitsunfalls erfüllen kann.

\section{Versicherungsschutz bei COVID-19 für Personen, die im Gesundheitsdienst und in der Wohlfahrtspflege tätig sind}

Die Leistungspflicht der Unfallversicherung setzt die Feststellung eines Arbeitsunfalls oder einer Berufskrankheit voraus. Die rechtliche Beurteilung über das Vorliegen einer Berufskrankheit oder eines Arbeitsunfalls bei COVID-19 erfolgt nach denselben Grundsätzen wie bei anderen viralen Infektionen auch.

Die Erkrankung infolge einer nachweislich beruflich erworbenen Infektion mit dem Coronavirus SARS-CoV-2 wird als Berufskrankheit anerkannt, soweit die rechtlichen Voraussetzungen im Einzelfall vorliegen. Wenn bereits eine Anerkennung als Berufskrankheit möglich ist, bedarf es keiner weiteren Prüfung eines Arbeitsunfalls.

\section{Merke}

Als Berufskrankheit kommt bei COVID-19 eine Berufskrankheit (BK) nach der Nr. 3101 der Anlage 1 zur Berufskrankheitenverordnung (BKV) in Betracht.

Diese Berufskrankheit ist wie folgt definiert:

„Infektionskrankheiten, wenn der Versicherte im Gesundheitsdienst, in der Wohlfahrtspflege oder in einem Laboratorium tätig oder durch eine andere Tätigkeit der Infektionsgefahr in ähnlichem Maße besonders ausgesetzt war".

Aufgrund dieses Wortlauts können COVID-19 Erkrankungen nur dann unter die BK-Nr. 3101 subsumiert werden, wenn sie bei versicherten Personen auftreten, die infolge der Ausübung ihrer beruflichen Tätigkeit in einem der folgenden, ausdrücklich genannten Bereiche einer gegenüber der allgemeinen Bevölkerung wesentlich erhöhten Infektionsgefahr ausgesetzt waren:

- 1. Alternative: Gesundheitsdienst,

- 2. Alternative: Wohlfahrtspflege,

- 3. Alternative: Laboratorium oder

- 4. Alternative: Bei Tätigkeiten, bei denen versicherte Personen der Infektionsgefahr in einem ähnlichen Maße, wie bei Tätigkeiten nach der 1. bis 3. Alternative besonders ausgesetzt waren.

Der Begriff „Gesundheitsdienst“ erfasst alle Tätigkeiten und Einrichtungen, bei denen die Sorge um die Gesundheit den 
Hauptzweck bildet. Erfasst werden auch abgrenzbare Unternehmensteile, soweit diese gesundheitsdienstliche Zwecke verfolgen, wie z. B. werksärztliche Abteilungen; ebenso Abteilungen einer Behörde mit entsprechender Zwecksetzung. Zum Gesundheitsdienst im Sinne der BK 3101 gehören insbesondere:

- Krankenhäuser,

- Medizinische Rehabilitationseinrichtungen,

- Entbindungseinrichtungen,

- Arzt- und Zahnarztpraxen,

- Apotheken,

- Physio-, Ergotherapieeinrichtungen o. ä.,

- Desinfektionsabteilungen und -betriebe,

- Krankentransport und Rettungsdienste sowie

- Pflegedienstleistungen.

Von der BK-Nr. 3101 werden neben den Laboratorien für wissenschaftliche und medizinische Untersuchungen und Versuche alle sonstigen Laboratorien erfasst, soweit es sich um Einrichtungen mit besonderen Infektionsgefahren handelt (z. B. auch Laboratorien für Zahntechnik). Dort Tätige müssen entweder mit Kranken unmittelbar in Berührung kommen oder mit Stoffen umgehen, die kranken Menschen zur Untersuchung entnommen wurden.

Auf die besonderen Voraussetzungen der von der BK-Nr. 3101 erfassten Tätigkeiten in der Wohlfahrtspflege und nach der oben angesprochenen vierten Alternative wird an späterer Stelle, im zweiten Teil dieses Beitrags, eingegangen.

Zum Kreis der Personen, die in der Unfallversicherung pflichtversichert sind, zählen insbesondere Beschäftigte, also Arbeitnehmerinnen und Arbeitnehmer. Im Bereich der Gesundheitsberufe unterliegen daher z. B. alle Personen, die in einem Krankenhaus, einer Arztpraxis, einem Laboratorium oder in der Pflegebranche auf der Grundlage eines Arbeitsvertrags angestellt sind, unmittelbar dem Schutz in der Unfallversicherung, ohne dass es hierfür eines Antrags bedarf.

Gleiches gilt auch für folgende Personen:

- unentgeltlich, insbesondere ehrenamtlich im Gesundheitswesen oder der Wohlfahrtspflege Tätige;

- selbständig im Gesundheitswesen Tätige, soweit sie nicht ausdrücklich durch das Gesetz von der Versicherungspflicht befreit sind, und

- ehrenamtlich im Rettungswesen Tätige.

Auch wenn selbständig Tätige im Gesundheitsdienst und in der Wohlfahrtspflege grundsätzlich von der gesetzlichen Pflichtversicherung erfasst werden, hat der Gesetzgeber einige Berufsgruppen ausdrücklich von der Versicherungspflicht befreit. Hierzu zählen neben einigen anderen Gesundheitsberufen u. a. auch die selbständig tätigen Ärztinnen und Ärzte, Zahnärztinnen und Zahnärzte sowie Apothekerinnen und Apotheker. Diese haben aber die Möglichkeit, sich auf Antrag freiwillig zu versichern. Dies gilt nicht nur für niedergelassene selbständige Ärztinnen und Ärzte, sondern bspw. auch für Ärztinnen und Ärzte, die auf Honorarbasis in einer Klinik arbeiten, und für angestellte Ärztinnen und Ärzte, soweit sie eine selbständige Nebentätigkeit ausüben. Gerade in Hinblick auf die mit den beruflichen Tätigkeiten im Gesundheitsdienst verbundenen Infektionsrisiken ist der Abschluss einer freiwilligen Versicherung zu überlegen.
Weitergehende aktuelle Informationen zum Kreis der versicherten Personen, insbesondere zum Versicherungsschutz von Personen, die unentgeltlich bzw. ehrenamtlich im Rahmen der Bekämpfung der Corona-Pandemie tätig werden (z. B. Studierende der Medizin), werden u. a. auf der Internet-Seite der BGW bereitgestellt: https://www.bgw-online.de/DE/Home/Branchen/News/ Coronavirus_node.html.

Eine der wichtigsten Aufgaben der Unfallversicherung besteht in der Verhütung von Arbeitsunfällen und Berufskrankheiten. Daher wird von den Unfallversicherungsträgern großer Wert auf die Einhaltung des Arbeitsschutzes und der erforderlichen Hygienemaßnahmen zur Vermeidung von Infektionen gelegt. Zu Beginn der Pandemie trat gehäuft die Situation ein, dass die Versorgung der medizinischen Einrichtungen mit der notwendigen Ausstattung zum Schutz vor einer Infektion mit dem Coronavirus SARS-CoV-2 (z. B. geeignete Atemschutzmasken) nicht immer sichergestellt werden konnte. Sollte die notwendige Schutzausrüstung in einem Unternehmen nicht vorhanden oder von den versicherten Personen nicht genutzt worden sein, schließt dies den Schutz durch die Unfallversicherung nicht aus.

Neben einer Tätigkeit in einem der in der BK-Nr. 3101 ausdrücklich genannten Bereiche setzt diese Berufskrankheit nach den rechtlichen Beweisregeln den Nachweis einer Infektionskrankheit im sog. Vollbeweis voraus.

\section{Merke}

Eine Antikörperbildung nach Aufnahme eines Infektionserregers ohne jegliche klinischen Symptome stellt keine Infektionskrankheit im Sinne des Berufskrankheitenrechts dar.

Die klinischen Symptome müssen allerdings keineswegs so ausgeprägt sein, dass damit eine ärztliche Behandlungsbedürftigkeit oder die Notwendigkeit einer Medikamentenversorgung verbunden ist. Es reichen somit auch geringfügige klinische Symptome, wie sie im Zusammenhang mit dem SARS-CoV-2 Erreger häufig festzustellen sind, zur Erfüllung des Krankheitsbegriffs bzw. für eine Anerkennung als Berufskrankheit aus. Grundsätzlich ist die Anerkennung einer Berufskrankheit nicht daran gebunden, dass dem Versicherten auch leistungsrechtliche Ansprüche (z. B. ärztliche Behandlung, Versorgung mit Arznei- oder Heilmitteln, stationäre Behandlung, Rente wegen Minderung der Erwerbsfähigkeit) zustehen. Die formale Anerkennung gibt dem Versicherten die Sicherheit, dass im Falle eines späteren Auftretens von Krankheitssymptomen das Vorliegen der Berufskrankheit dem Grunde nach bereits feststeht.

Da die Unfallversicherung allein für die Verwirklichung eines versicherten (i. d. R. beruflichen) Infektionsrisikos einzustehen hat, ist immer der Nachweis eines Verursachungszusammenhangs zwischen dem versicherten erhöhten Infektionsrisiko und dem Eintritt der Erkrankung festzustellen. Sein Nachweis ist erbracht, wenn die berufliche Verursachung überwiegend wahrscheinlich ist. Dieser Kausalzusammenhang ist bei der BK-Nr. 3101 in der Regel gegeben, wenn die versicherte Person während des in Frage kommenden Ansteckungszeitraums bei ihrer versicherten Tätigkeit 
- Kontakt zu mindestens einer nachgewiesenen Infektionsquelle (z. B. Patienten, Kollegen, Besucher, Untersuchungsmaterialien usw.) hatte,

- nach der Art des Kontaktes eine Infektionsübertragung dabei konkret möglich war und

- Umstände aus dem unversicherten Bereich oder eine ausgeprägte Ubiquität des Infektionserregers einem Schluss auf die Wahrscheinlichkeit des Zusammenhangs mit der versicherten Tätigkeit nicht entgegenstehen. [12, 13].

Da die Symptome bei COVID-19 unspezifisch sind, ist der Nachweis einer Infektion der in Frage kommenden Indexperson grundsätzlich durch einen zeitnahen Erreger-Nachweistest zu erbringen. Wurde dieser Test nicht durchgeführt, erbringt aber ein später durchgeführter Antikörper-Test den Nachweis einer durchgemachten Infektion, kommt es auf die Umstände des Einzelfalles an, ob die betreffende Person als Indexperson geeignet ist. Kriterien sind dabei insbesondere die Art der Krankheitssymptome zum Zeitpunkt der zu beurteilenden Kontaktsituation und das zeitliche Intervall zwischen dem Kontakt und dem Antikörpertest.

Lässt sich bezüglich der Kontakte im versicherten Umfeld ein Verdacht, dass es sich um infektiöse Quellen handelt, nicht bestätigen, kann auf den bloßen Verdacht allein die Wahrscheinlichkeit eines Kausalzusammenhangs nicht gestützt werden. Die Häufigkeit und Intensität von Kontakten zu infektionsverdächtigen Quellen, kann aber wesentlich für die Prüfung sein, ob eine Beweiserleichterung wegen einer durch die Art der konkret ausgeübten Tätigkeit bedingten besonders erhöhten Infektionsgefahr in Frage kommt. Auch ohne konkreten Nachweis von Kontakten mit einer nachgewiesenen Infektionsquelle kann nämlich der Kausalzusammenhang bei Beschäftigten im Gesundheitsdienst wahrscheinlich sein, soweit Versicherte während der in Frage kommenden Ansteckungszeit einer besonderen über das normale Maß hinausgehenden Infektionsgefahr ausgesetzt waren. Zur Konkretisierung einer in diesem Sinne für die Wahrscheinlichkeit der Kausalität ausreichenden Gefahrerhöhung ist zwischen der Verbreitung der Infektionskrankheit und dem Übertragungsweg zu unterscheiden. So kann z. B. ein größeres Ausbruchsgeschehen innerhalb eines Betriebs unter Berücksichtigung der konkreten Verhältnisse im Tätigkeitsbereich der Betroffenen im Einzelfall zu einem erleichterten Nachweis der beruflichen Verursachung führen.

Hinsichtlich des Übertragungsweges von Coronavirus SARSCoV-2 besteht eine Vergleichbarkeit mit dem Mycobacterium tuberculosis. Es bietet sich bei COVID-19 daher an, in Anlehnung an die für die Tuberkuloseinfektion vorliegenden Erkenntnisse bei bestimmten Tätigkeiten bzw. Bereichen von einer besonderen über das normale Maß hinausgehenden Infektionsgefahr auszugehen, die zur Anwendung einer Beweiserleichterung führen kann [14].

\section{INFO}

Tätigkeiten/Bereiche, die zu einer Beweiserleichterung führen können

- Klinik-Abteilungen, in denen Patienten mit COVID-19 behandelt werden (z. B. Infektionsstationen)

- ambulante oder stationäre Untersuchungseinheiten für SARS-CoV-2-Infektionen
- intensivmedizinischen Behandlungseinheiten,

- Labore, die Abstriche auf SARS-CoV-2 untersuchen,

- Notfallintubation,

- Bronchoskopie,

- Provokation von Hustenreiz z. B. bei Nasen-Rachen-Abstrichen

- Betreuung von Hochrisikogruppen (z. B. Menschen aus Ländern mit hoher Inzidenz, Menschen, die Kontaktsperren und Distanzgebote nicht einhalten oder nicht einhalten können),

- Auslandseinsätze in Gebieten mit hoher Inzidenz,

- Unter Berücksichtigung der jeweiligen besonderen Rahmenbedingungen bei der Pflege oder vergleichbaren Tätigkeiten in der klinischen Geriatrie, sofern ein enger Kontakt bei einem hohen Anteil pflegebedürftiger Personen festzustellen ist.

Diese Aufzählung ist nicht abschließend. Sie dient lediglich der Orientierung.

Erkenntnisse aus epidemiologischen Untersuchungen zu einer erhöhten Erkrankungsinzidenz und -prävalenz mit COVID-19 bei bestimmten Tätigkeiten im Gesundheitsdienst werden erst zukünftig vorliegen können. Sofern sich aufgrund der umfangreichen Durchführung von Antikörper-Tests ergibt, dass von weitaus höheren Infektionszahlen auszugehen ist, muss die Reichweite von Beweiserleichterungen gegebenenfalls neu justieret werden; es ist nicht ausgeschlossen, dass dann auch für weitere vor allem im Gesundheitsdienst tätige Berufsgruppen eine Beweiserleichterung begründbar ist.

Wurde COVID-19 als Berufskrankheit (oder als Arbeitsunfall) anerkannt, erbringt der Unfallversicherungsträger folgende Leistungen, soweit diese im jeweiligen Einzelfall geboten sind:

\section{a) Heilbehandlung}

Die gesetzliche Unfallversicherung hat die Aufgabe, die Gesundheit der Versicherten mit allen geeigneten Mitteln möglichst vollständig wiederherzustellen. Daher übernimmt sie u. a. die Kosten für die

- medizinische Erstversorgung,

- stationäre und ambulante ärztliche Behandlung,

- Versorgung mit Arznei-, Verband-, Heil- und Hilfsmitteln,

- häusliche Krankenpflege sowie

- Leistungen zur medizinischen Rehabilitation.

Im Falle der Arbeitsunfähigkeit infolge eines Versicherungsfalles wird ein Verletztengeld gezahlt (nach Ablauf der Entgeltfortzahlung durch den Arbeitgeber), um den Verdienstausfall auszugleichen. Dieses Verletztengeld wird auch während der Dauer einer medizinischen Rehabilitation gezahlt.

b) Leistungen zur beruflichen und sozialen Teilhabe

Der Unfallversicherungsträger versucht die Rückkehr an den bisherigen Arbeitsplatz mit allen geeigneten Mitteln zu ermöglichen. In Abstimmung mit den Versicherten und dem Arbeitgeber werden dafür alle notwendigen Maßnahmen getroffen. 


\section{c) Entschädigung durch Rente}

Nicht immer sind Heilbehandlung und Reha-Maßnahmen so erfolgreich, dass Betroffene wieder uneingeschränkt am Erwerbsleben teilnehmen können. In solchen Fällen zahlen die Unfallversicherungsträger eine Rente. Voraussetzung ist eine andauernde Minderung der Erwerbsfähigkeit (MdE) von mindestens 20 Prozent infolge des Versicherungsfalls.

d) Pflegeleistungen

Personen, die infolge des Versicherungsfalls so hilflos sind, dass sie in erheblichem Umfang fremder Hilfe bedürfen, erhalten Pflegegeld bzw. Haus- bzw. Heimpflege.

\section{e) Leistungen an Hinterbliebene}

Kommt es zu einem tödlichen Verlauf der Erkrankung, dann sichert die gesetzliche Unfallversicherung die Hinterbliebenen mit finanziellen Leistungen ab.

Dazu gehören insbesondere:

- Sterbegeld,

- Überführungskosten, wenn der Tod nicht am Ort der ständigen Familienwohnung des Versicherten eingetreten ist,

- Hinterbliebenenrenten.

Weiterführende Informationen zu den einzelnen Leistungen (insbesondere auch zu ihrem Umfang) sind auf der Homepage der Deutschen Gesetzlichen Unfallversicherung (DGUV) zu finden:

https://www.dguv.de/de/reha_leistung/index.jsp

\section{Wann ist COVID-19 der Unfallversicherung zu melden?}

\begin{abstract}
Merke
Ärztinnen und Ärzte sowie Zahnärztinnen und Zahnärzte sind nach § 202 SGB VII verpflichtet, den begründeten Verdacht auf eine Berufskrankheit dem Träger der Unfallversicherung oder dem staatlichen Gewerbearzt/Landesgewerbearzt zu melden.
\end{abstract}

Die Anzeigen haben formalisiert zu erfolgen (Formular unter https://www.dguv.de/bk-info/service/index.jsp); sie bedürfen nicht der Einwilligung der betroffenen Patienten. Die gemeldete Person ist über den Adressaten und Inhalt der Verdachtsanzeige zu informieren, zweckmäßigerweise wird ihr eine Kopie mitgegeben oder geschickt. Die Betroffenen können auch selbst eine Berufskrankheit anzeigen. Dies entbindet die Ärztin / den Arzt aber nicht von der Anzeigepflicht [15].

Der Verdacht auf eine Berufskrankheit ist im Sinne des $\S 202$ SGB VII dann begründet, wenn der Ärztin/dem Arzt ernsthafte, konkrete Anhaltspunkte für deren Bestehen hat. Die reine Vermutung ist nicht ausreichend; die völlige Gewissheit aber nicht erforderlich für eine Meldung. Die hier dargestellten Maßstäbe sollen Ärztinnen und Ärzte bei einer sachgerechten Einschätzung unterstützen.

Im Falle einer Infektion mit dem Coronavirus SARS-CoV-2 kommt - wie bereits oben, in Teil 1 dargelegt - eine Berufskrankheit nach Nr. 3101 in Betracht. Diese findet nach ihrem Tatbestand nur Anwendung auf Infektionskrankheiten von versicherten Personen, die im Gesundheitsdienst (1. Alternative), in der Wohl- fahrtspflege (2. Alternative), in einem Laboratorium (3. Alternative) tätig sind oder durch eine andere Tätigkeit der Infektionsgefahr in ähnlichem Maße besonders ausgesetzt waren (4. Alternative). Andere Tätigkeiten werden von der BK-Nr. 3101 nicht erfasst; eine Meldepflicht nach § 202 SGB VII besteht dann nicht, selbst wenn deutliche Hinweise dafür vorliegen, dass sich die Infektion während der versicherten Tätigkeit ereignet hat. Es ist dann aber ggf. an die Möglichkeit eines Arbeitsunfalls zu denken (siehe hierzu Teil 3 dieses Beitrags).

Während auf die Tätigkeitsbereiche Gesundheitsdienst und Laboratorien bereits im ersten Teil eingegangen wurde, bedarf es hier noch Ausführungen zur Wohlfahrtspflege sowie zur vierten Alternative (Tätigkeiten mit einer Infektionsgefahr in ähnlichen Maße).

Unter Wohlfahrtspflege wird die zum Wohle der Allgemeinheit und nicht des Erwerbs wegen ausgeübte Betreuung von gesundheitlich, sittlich oder wirtschaftlich gefährdeten Menschen verstanden. Ein wesentlicher Aufgabenbereich der Wohlfahrtspflege ist die Verhütung oder Beseitigung von Notständen und sozialen Gefährdungen. Unter dem Aspekt der sozialen Hilfen werden aber auch Förderangebote vorgehalten, die sich nicht auf eine besondere Bedürftigkeitslage beziehen, sondern entsprechende allgemeine Förderangebote des Staates aus allgemeinen sozialen Gründen ergänzen oder ersetzen. Typischen Handlungsfelder der Wohlfahrtspflege finden sich in der

- Jugendhilfe (z. B. Kindertageseinrichtungen, Jugendwohnheime, Jugendberatungsstellen),

- Familienhilfe (z. B. Beratungsstellen für Ehe-, Familien-, Erziehungs- und Lebensfragen, Familientreffs, Frauenhäuser),

- Altenhilfe (z. B. vollstationäre Altenpflegeeinrichtungen, Seniorenwohnheime, Seniorenwohnungen, betreutes Wohnen, ambulante Pflegedienste, Seniorentagesstätten, Seniorenbegegnungsstätten),

- Hilfe für behinderte Menschen oder Menschen mit psychischer Erkrankung (z. B. Rehabilitationseinrichtungen, Beratungs- und Betreuungsstellen, Tagesstätten für Menschen mit psychischer, geistiger oder körperlicher Erkrankung bzw. Behinderung; Werkstätten für Menschen mit Behinderung, Fahrdienste) und der

- Hilfe für Personen in besonderen sozialen Situationen (z. B. Migrationsberatungsstellen, Tafelbetriebe, Schuldnerberatungsstellen, betreutes Wohnen und Tagesstätten für Migranten, Wohnungslose, Suchtkranke und Haftentlassene, Seemannsheime, Bahnhofsmissionen, Suchdienste, Studentenwohnheime, Stadtteil-/Nachbarschaftszentren).

Die vierte Tatbestandsalternative der BK-Nr. 3101 beinhaltet keinen Auffangtatbestand für all jene Fälle, die nicht unter die genauer genannten Einrichtungen der ersten bis dritten Alternative einzuordnen sind. Der Zweck dieser Regelung besteht vielmehr darin, auch die Personen in den Versicherungsschutz für Infektionskrankheiten einzubeziehen, die durch eine andere Tätigkeit in ähnlichem Maße einer Infektionsgefahr besonders ausgesetzt sind. Im Gegensatz zur Infektionsgefährdung bei den drei übrigen Tatbestandsalternativen der BK-Nr. 3101 ist bei der vierten Alternative zu berücksichtigen, dass epidemiologische Studien über eine erhöhte Betroffenheit bestimmter Beschäftigtengruppen 
hinsichtlich COVID-19 bisher nicht vorliegen. Ausschlaggebend für die Zuordnung zur vierten Alternative der BK-Nr. 3101 ist die Art des physischen menschlichen Kontakts, der mit der versicherten Tätigkeit verbunden ist.

Dabei kommt es nicht allein darauf an, dass die Tätigkeit mit einem häufigen und/oder direkten Kontakt zu infizierten Personen verbunden ist bzw. sein kann.

Es ist darüber hinaus zu unterscheiden zwischen

- dem auftragsgemäßen oder dem mit der Arbeit verbundenen „Kontakt am Menschen“, worauf sich der Anwendungsbereich der BK-Nr. 3101 bezieht und

- dem situativen „Kontakt zu Menschen“.

Die Beschäftigung mit „Kontakt am Menschen“ umfasst Tätigkeiten, die bestimmungsgemäß

- mit einem unmittelbaren Körperkontakt (z. B. Masseure usw.) oder

- mit sog. „gesichtsnahen Tätigkeiten“ (z. B. Friseure, Kosmetiker, Optiker usw.)

verbunden sind. Das abstrakte Risiko einer aerogenen Infektion mit COVID-19 ist bei diesen Tätigkeiten zumindest hinsichtlich des Übertragungsweges vergleichbar mit dem Risiko der in den ersten beiden Alternativen der BK-Nr. 3101 genannten Tätigkeiten. In den übrigen Fällen - also bei Tätigkeiten mit einem situativen häufigen und direkten Kontakt „Kontakt zu Menschen“ kommt eine Prüfung anhand der rechtlichen Vorgaben für einen Arbeitsunfall in Betracht.

\section{COVID-19 als Arbeitsunfall}

Merke

Erfolgt eine Infektion mit dem Corona-Virus SARS-CoV-2 in

Ausübung einer Beschäftigung, kann eine sich daraus ergebende COVID-19-Erkrankung auf der Grundlage der aktuellen Erkenntnisse auch einen Arbeitsunfall darstellen.

Dies kommt dann in Betracht, wenn die Infektion nicht in einem Bereich erfolgte, der von der BK-Nr. 3101 erfasst wird. Dies betrifft Konstellationen, in denen sich der intensive und direkte Kontakt zu infizierten Personen nicht bestimmungsgemäß, sondern vielmehr situativ aus der versicherten Tätigkeit ergibt (Beispiel: Infektion eines Beschäftigten während einer dienstlich veranlassten Bahnreise bei einem anderen Fahrgast, der in unmittelbarer Nähe sitzt).

Auch die Anerkennung eines Arbeitsunfalls setzt in der Regel einen nachgewiesenen intensiven Kontakt mit einer infizierten Indexperson voraus. Maßgeblich sind dabei im Wesentlichen die Häufigkeit, die Dauer sowie die Intensität des Kontaktes. Lässt sich keine konkrete Indexperson ermitteln, kann - wie auch bei der Berufskrankheit - ein Ausbruchsgeschehen in einem Betrieb im Einzelfall zu einem erleichterten Nachweis der beruflichen Verursachung führen.

Im Gegensatz zu einer Berufskrankheit kommt die Anerkennung eines Arbeitsunfalls auch dann in Betracht, wenn die versicherte Person sich auf dem Weg zur oder von der Arbeit infiziert hat. In bestimmten Konstellationen kann auch eine Infektion beim Kantinenbesuch oder beim Aufenthalt in einer Gemeinschaftsunterkunft ausnahmsweise versichert sein, wenn die besondere Infektionsgefährdung dem unternehmerischen Verantwortungsbereich zugerechnet werden muss.

Grundsätzlich haben Ärztinnen und Ärzte, die/der keine Zulassung als Durchgangsarzt haben, die Betroffenen nach einem Arbeitsunfall anzuhalten, sich bei einem Durchgangsarzt vorzustellen, wenn die nachfolgenden Voraussetzungen vorliegen:

- die Erkrankung zu einer mehrtägigen Arbeitsunfähigkeit führt,

- die notwendige ärztliche Behandlung voraussichtlich über eine Woche andauert oder

- Heil- und Hilfsmittel zu verordnen sind.

Für Patientinnen und Patienten mit einer wahrscheinlich beruflich erworbenen Covid-19 Infektion, bei denen aus den oben genannten Gründen ausschließlich ein Arbeitsunfall in Betracht zu ziehen ist, haben sich die Unfallversicherungsträger jedoch auf ein anderes Vorgehen verständigt: Aus Gründen der Infektionsprävention ist eine Vorstellung beim Durchgangsarzt einzig zur Aufnahme der versicherungsrechtlich relevanten Daten nicht sinnvoll. Vor dem Hintergrund der besonderen Sachlage, Quarantäneauflagen und fachspezifischen Behandlung greift die übliche Vorstellungspflicht beim Durchgangsarzt hier nicht. In diesen Fällen erfolgt die Meldung an den Unfallversicherungsträger mit der Ärztlichen Unfallmeldung (F 1050). Sollten sich Betroffene dennoch bei einer Durchgangsärztin/einem Durchgangsarzt vorstellen, ist ein D-Bericht (F 1000) zu erstellen.

Eine gesetzliche Meldepflicht für Arbeitsunfälle besteht für Arbeitgeber, wenn eine versicherte Person durch einen Unfall getötet oder so verletzt wurde, dass sie mehr als drei Tage arbeitsunfähig ist (§193 SGB VII). Die Drei-Tages-Frist beginnt am Tag nach dem Unfall und umfasst alle Kalendertage.

KERNAUSSAGEN

- COVID-19 kann Berufskrankheit oder Arbeitsunfall sein.

- Jeder begründete Verdacht auf eine Berufskrankheit ist für die Ärztin/den Arzt meldepflichtig.

Interessenkonflikte

DN und UO fertigen Gutachten über Berufskrankheiten für Unfallversicherungsträger und Sozialgerichte an.

SB und MW sind Mitarbeiter eines Unfallversicherungsträgers, der von COVID-19-Fällen besonders betroffenen Berufsgenossenschaft für Gesundheitsdienst und Wohlfahrtspflege. AN ist Inhaber einer Stiftungsprofessur der Berufsgenossenschaft für Gesundheitsdienst und Wohlfahrtspflege.

Literatur

[1] Lietz J, Westermann C, Nienhaus A et al. The Occupational Risk of Influenza A (H1N1) Infection among Healthcare Personnel during the 2009 Pandemic: A Systematic Review and Meta-Analysis of Observational Studies. PLoS One 2016; 11 (8): e0162061 
[2] Chowell G, Abdirizak F, Lee S et al. Transmission characteristics of MERS and SARS in the healthcare setting: a comparative study. BMC 2015; 13: 210

[3] Hunter E, Price DA, Murphy E et al. First experience of COVID-19 screening of health-care workers in England. Lancet 2020; 395: e77-e78

[4] Zhang M. Protecting healthcare workers in China during the coronavirus outbreak. BMJ opinion 2020. https://blogs.bmj.com/bmj/2020/02/14/ min-zhang-protecting-healthcare-workers-china-coronavirus-outbreak/

[5] Zhang Z, Liu S, Xiang M et al. Protecting healthcare personnel from 2019nCoV infection risks: lessons and suggestions. Front Med 2020; 14 (2): 229-231

[6] Istituto Superiore di Sanità. Integrated surveillance of COVID-19 in Italy: 10 April 2020. https://www.epicentro.iss.it/en/coronavirus/bollettino/In fografica_10aprile\%20ENG.pdf

[7] Koh D. Occupational risks for COVID-19 infection. Occupational Medicine 2020; 70: 3-5

[8] Rothe C, Schunk M, Sothmann P et al. Transmission of 2019-nCoV infection from an asymptomatic contact in Germany. N Engl J Med 2020; 382: 970-971

[9] Wander PL, Orlov M, Merel SE et al. Risk factors for serious/critical COVID-19 illness in health care workers: Too many unknowns. Infect Control Hosp Epidemiol 2020: 1-2. doi:10.1017/ice.2020.178
[10] RKI Täglicher Lagebericht des RKI zur Coronavirus-Krankheit-2019 (COVID-19) 14.12.2020. https://www.rki.de/DE/Content/InfAZ/N/Neu artiges_Coronavirus/Situationsberichte/Dez_2020/2020-12-14-de.pdf? _blob=publicationFile

[11] Kersten JF, Nienhaus A, Schneider S et al. Tuberculosis among Health Workers - A Secondary Data Analysis of German Social Accident Insurance Data from 2002-2017. Int J Environ Res Public Health 2020; 17 (5): 1564. doi:10.3390/ijerph170 1564

[12] Brandenburg S. Rechtliche Voraussetzungen für die Anerkennung und Entschädigung von Hepatitis B- und C-Infektionen als Berufskrankheit S. 199-207. In: Selmair H, Manns MP Virushepatitis als Berufskrankheit Ein Leitfaden zur Begutachtung. 3. Aufl. Landsberg: Ecomed; 2007

[13] BSG, Urteil vom 2.4.2009, Az. B 2 U 7/08 R.

[14] Brandenburg S, Woltjen M. Rechtliche Voraussetzungen für die Anerkennung und Entschädigung der Tuberkulose als Berufskrankheit, S. 91 98. In: Nienhaus A, Brandenburg S, Teschler H Tuberkulose als Berufskrankheit - Ein Leitfaden zur Begutachtung und Vorsorge. 4. Aufl. Landsberg: Ecomed; 2017

[15] Nowak D, Brandenburg S. Zusammenhangsbegutachtung von Berufs krankheiten - die Rolle des Arztes. Dtsch Med Wochenschr 2019; 144 1487-1495 